\title{
Novel method for determination of gas properties and flow speed
}

\author{
Christoph J. Hepp ${ }^{1}$, Florian T. Krogmann ${ }^{1}$, Gerald A. Urban ${ }^{2}$ \\ ${ }^{1}$ Innovative Sensor Technology IST AG, Stegruetistrasse 14, 9642 Ebnat-Kappel, Switzerland, \\ christoph.hepp@ist-ag.com \\ ${ }^{2}$ Laboratory for Sensors, Department of Microsystems Engineering - IMTEK, University of Freiburg, \\ Georges-Koehler-Allee 103, 79110 Freiburg, Germany
}

\begin{abstract}
:
A novel method to determine the thermal gas parameters (thermal conductivity and the product of density and specific heat capacity) and flow speed are discussed in this contribution. We developed a procedure to use different time independent excitation modes for this purpose in contrast to other research groups who are using AC excitation. However, certain conditions have to be respected, they are examined in detail. The sensor design is based on a calorimetric flow sensor, thus a one chip solution is sufficient. Theoretical investigations and experimental data are presented.
\end{abstract}

Key words: Thermal Flow Sensor, Time independent excitation, Thermal properties, Flowing fluid.

\section{Introduction}

In 1973, the first micro machined thermal flow sensor was developed by van Putten [1]. Since that date extensive research has been carried out in this field by several research groups and it is still an ongoing topic. The main advantage of thermal sensors compared to other flow measurement principles are low power consumption, fast response time and an outstanding long-term stability. For a long time, the research focus was in this field. Thermal flow sensors are well established in the market. Typical applications are in process control or medical engineering.

The working principle of thermal flow sensors can be classified in three categories: anemometers, calorimeters and time of flight sensors [2].

Anemometers feature at least one element, used simultaneously as joule heating element and temperature sensor. A widely used embodiment - however not fabricated micro machined - is still the hot wire anemometer. Fabricated with micro machined technology, they are known as hot film anemometers. Common evaluation electronics are the constant current (CCA) and the constant temperature anemometer (CTA).

Calorimetric flow sensors usually consist of one centered heating element, which is surrounded by two temperature sensors; one located upstream and one downstream. The temperature difference between the up- and downstream element is measure for the mass flow.

Time of flight sensors are equipped with one heating element and a temperature sensor, which is located downstream. The time a head pulse needs from the heater to the temperature sensor is a measure for the flow rate.

Beside flow rate, thermal flow sensors are sensitive to the gas and therewith to the thermal properties, where they are applied in. Hence, they need to be calibrated to the specific gas or to the application respectively. Table 1 shows thermal properties of several gases. This table depicts how different these properties can be.

Due to this drawback, intensive research is done in the field towards a medium independent thermal flow sensor $[3,4]$. On the other hand the simultaneous detection of flow speed and the gas will offer new application fields. So far, it was not possible to use thermal flow sensors in applications, where gas type and flow speed are changing simultaneously.

In this contribution, we present a novel method to determine thermal gas parameters and flow speed using time independent excitation modes. It is presented under which conditions we can measure the thermal gas parameters thermal conductivity and the product of specific heat capacity and density and beyond with which excitation mode flow velocity can be 
extracted. For this purpose a one single chip solution is used.

Tab. 1: Thermal gas properties at a pressure of $1 \mathrm{bar}$ and a temperature of $T=20^{\circ} \mathrm{C}$ and $T=25^{\circ} \mathrm{C}^{*}$ [5].

\begin{tabular}{|c|c|c|c|}
\hline Gas type & $\begin{array}{c}\text { Thermal } \\
\text { conductivity } \\
{[\mathrm{mW} /(\mathrm{m} \cdot \mathrm{K})]}\end{array}$ & $\begin{array}{c}\text { Specific } \\
\text { heat } \\
\text { capacity } \\
{[\mathrm{J} /(\mathrm{g} \cdot \mathrm{K})]}\end{array}$ & $\begin{array}{c}\text { Density } \\
{\left[10^{-3}\right.} \\
\left.\mathrm{g} / \mathrm{cm}^{3}\right]\end{array}$ \\
\hline $\mathrm{Ne}^{*}$ & 49.0 & 1030 & 0.828 \\
\hline $\mathrm{Ar}$ & 17.8 & 521.5 & 1.603 \\
\hline $\mathrm{CO}_{2}$ & 16.8 & 825 & 1.773 \\
\hline $\mathrm{O}_{2}$ & 26.5 & 919.9 & 1.284 \\
\hline $\mathrm{N}_{2}$ & 26.0 & 1041 & 1.123 \\
\hline Air & 26.4 & 1007 & 1.161 \\
\hline $\begin{array}{c}\mathrm{N}_{2}(\text { with } \\
\left.1.5 \mathrm{C}_{\mathrm{p}}\right)\end{array}$ & 26.0 & $1.5^{*} 1041$ & 1.123 \\
\hline $\begin{array}{c}\mathrm{N}_{2} \text { (with } \\
1.5 \rho)\end{array}$ & 26.0 & 1041 & $1.5^{\star} 1.123$ \\
\hline
\end{tabular}

\section{Sensor Description}

The design is based on a calorimetric flow sensor. Figure 1 shows the top view of the sensor's membrane structure.

Foturan glass serves as substrate material in order to enable a robust handling. The membrane area has an oval shape with an area of $2 \mathrm{~mm} \times 1 \mathrm{~mm}$. Polyimide is chosen as membrane material due to its low thermal conductivity. The chip includes two temperature sensors located up- and downstream from the centered joule heating element. They are designed as resistance temperature detectors (RTD). All elements are made of platinum due to the well-defined TCR and resistance to temperature behavior.

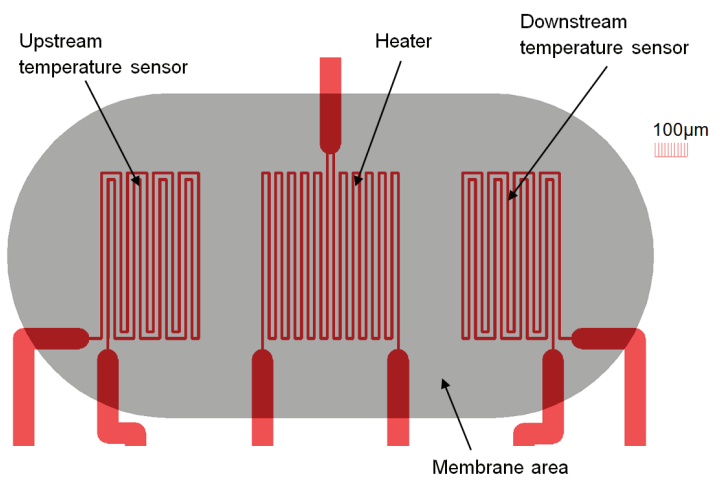

Fig. 1. Top view of sensor's membrane area. Upand downstream description marks the flow direction according to the heating element.

\section{Simulation Model}

The numerical model is set up in COMSOL Multiphysics. COMSOL Multiphysics offers the possibility to couple different physical phenomena. The model combines the Heat Transfer (ht-mode) and the incompressible Navier-Stokes equation (spf-mode) equation in stationary domain. Based on the setting up multi physics in the model, changes in thermal properties due to temperature variations are taken into account.

The geometry is designed in three dimensions and symmetrical to the z-axes. The model is shown in Figure 2. The flow channel has a cross section of $1 \mathrm{~mm}^{2}$. Inside the channel the average flow speed is varied between $10^{-4}$ and $1.5 \mathrm{~m} / \mathrm{s}$. Different gases are investigated (see Table 1).

Heater (centered element) and temperature sensors (located up- and downstream of the heater) are embedded in the middle of the membrane using the highly conductive layer feature. The thermal properties of these areas are calculated according to the area percentage of platinum and polyimide; because these areas are designed as RTD's with meander lines according to Figure 1 for fabrication. The cavity underneath the elements is filled with air independent of the gas in the channel. The heater is operated in constant power mode (DC) with a heating power of $12 \mathrm{~mW}$.

The downstream element and the heater temperature are investigated for various gases and flow speed as output signals.

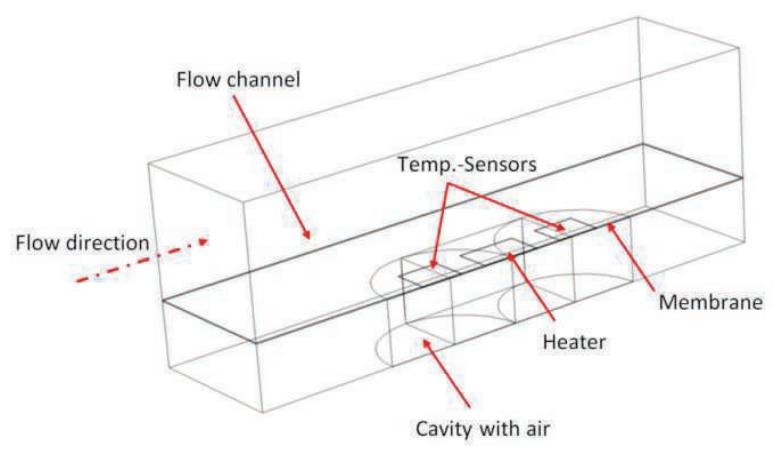

Fig. 2. 3D FEM-Simulation model set up in COMSOL Multiphysics. Model combines Heat Transfer and Laminar Flow mode.

\section{Simulation results}

The flow profile inside the channel is fully developed and laminar independent of the gas due to the chosen boundary conditions.

Figure 3 shows the temperature variation of the heating element versus flow speed for various gases (ambient temperature subtracted). As can be seen the heating temperature decreases with an increase of flow speed. A gas with a 
high thermal conductivity leads to a lower heater temperature. The value at zero flow depends only on the thermal conductivity; however the cooling rate is affected as well from density multiplied by specific heat capacity. The figure indicates that the product of specific heat capacity $\left(c_{p}\right)$, density $(\rho)$ and flow speed $(v)$ is always constant. It means that gases with the same value in thermal conductivity and the same value in this product $\left(c_{p} \bullet \rho \bullet v\right)$, having the same heater temperature.

Hence, it seems to be possible to calculate specific heat capacity times density, if thermal conductivity and flow speed are known.

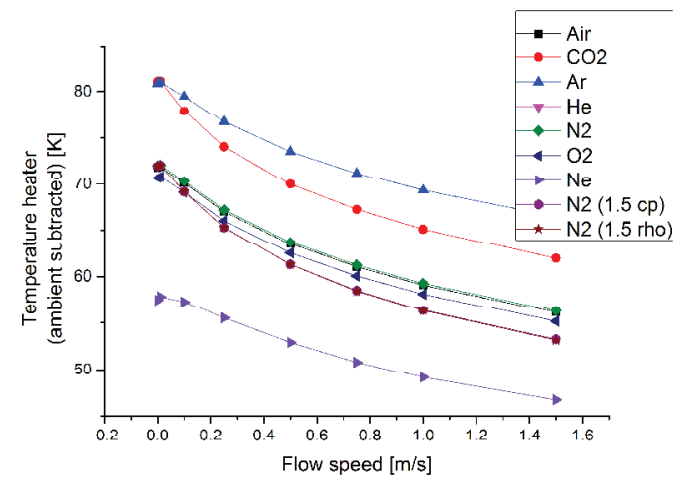

Fig. 3. Temperature of heating element versus flow speed for different gases. - Simulation results.

The temperature of the downstream element (ambient temperature subtracted) as function of flow speed is depicted in Figure 4 by using constant power excitation. The temperature is increasing by an increasing flow speed. After a certain flow value, the temperature remains nearly constant; however flow speed is still increased. This region is defined as flow independent in context of the work, because the temperature variation related to the maximum is less than $2 \%$ in the flow range between 0.3 to $1.1 \mathrm{~m} / \mathrm{s}$. Furthermore, thermal conductivity seems to be the dominant parameter, because the temperature is almost equal for the gases argon and carbon dioxide. Temperature is rising by a decreasing thermal conductivity (compare neon to carbon dioxide).

They have nearly the same value in thermal conductivity but differ in the other thermal parameters as density and specific heat capacity. A change in these two values ( $c_{p}$ and $\rho)$ is affecting the size of the flow independent region, but there influence will be neglected in a first approach.

Thus, the temperature in this flow independent region is sensitive to the gas type, but not affected from the flow speed. It seems to be possible to detect thermal conductivity in this region.

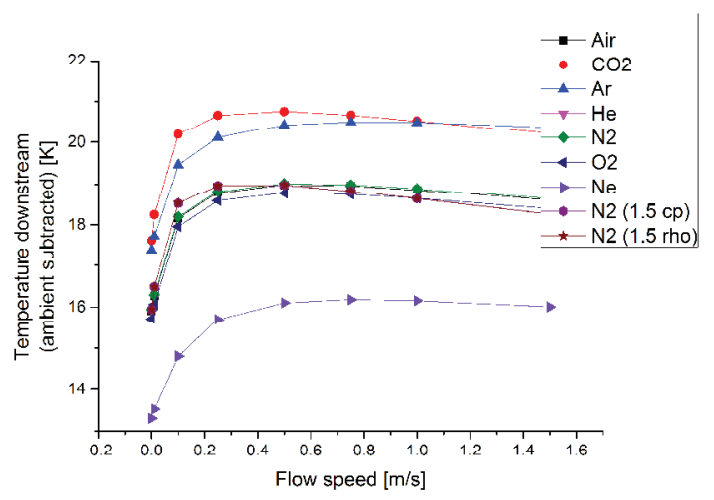

Fig. 4. Temperature of the downstream element versus flow speed for different gases. - Simulation results.

\section{Measurement Setup}

Measurements should verify the previous carried out simulations. As bottom of the flow channel serves a printed circuit board (PCB) with a cavity. Inside this cavity the chip is mounted and electrically connected with a bonding process. Above the PCB a flow channel is mounted with a cross section of $2 \mathrm{~mm}^{2}$. The length of the channel is $40.8 \mathrm{~mm}$ and the chip is placed in the middle. Mass flow controllers (MFCs) are used to adjust the volume flow. The volume flow is varied between 0 and $200 \mathrm{sccm}$.

\section{Measurement Principle}

The heating element can be operated either in constant temperature or constant power mode, therefore a controller is implemented in a LABVIEW program. Right now, the controller needs to be manually switched between the different excitation modes. The power consumption of the heating element is $12 \mathrm{~mW}$ in constant power mode. The constant temperature mode sets a temperature difference between heating element and ambient to $75 \mathrm{~K}$.

The current through the downstream element is $0.1 \mathrm{~mA}$ to avoid self heating effects of the resistor. The voltage drop is acquired with a data acquisition card (NI USB-6225). The resistance is calculated by the Ohm's law and afterwards the temperature by:

$$
R(T)=R_{0}\left(1+\alpha \cdot\left(T-T_{0}\right)\right)
$$

where $R(T)$ is the resistance at temperature $T$, $\alpha$ is the temperature resistance coefficient (TCR), $R_{0}$ is the resistance value at a defined temperature (usually at $0^{\circ} \mathrm{C}$ ) and $T_{0}$ is the temperature, where $R_{0}$ is defined. The TCR is obtained by an electrical characterization (resistance to temperature behavior). 
The temperature of the downstream element represents the output signal, which is investigated.

\section{Measurement results}

The output signal by constant power excitation is shown in Figure 5; in Figure 6 by constant temperature excitation.

The temperature progression of the downstream element corresponds to the simulation results by constant temperature - as well a flow independent region and a temperature increase by an increase in thermal conductivity are observed. The flow independent region according to the requirement in the simulations will be set between 25 and $125 \mathrm{sccm}$. By constant temperature excitation, the temperature is continuously rising and a function of flow speed and gas type. By knowing the gas type, it seems to be feasible to extract the flow speed signal by this output signal.

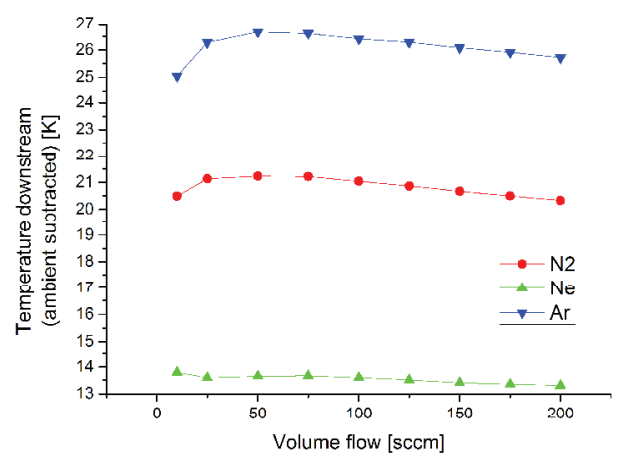

Fig. 5. Measurement result: Temperature of the downstream element as function of flow speed. Excitation mode: constant power.

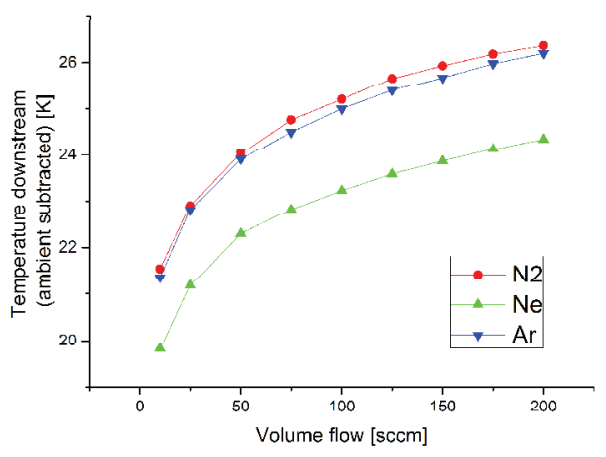

Fig. 6. Measurement result: Temperature of the downstream element as function of flow speed. Excitation mode: constant temperature.

\section{Discussion}

The thermal gas properties affect the heat distribution in the medium and therewith the temperature of every element. The flow independent region seems to be a variable of the sensor design, cooling of the heating element and a variation of the heat transfer by a change in flow speed.

The next working steps will be to precisely quantify the output signals regarding resolution etc. Furthermore, relations between the thermal parameters, sensor, design and the flow independent region have to be investigated.

\section{Conclusions}

A novel method for determining thermal gas parameters and flow speed has been presented. In contrast to other research groups, we developed a procedure to use time independent excitation modes for this purpose. The sensor layout is based on a calorimetric flow sensor. Platinum is used as heating element and RTDs to measure the temperature up- and downstream on membrane.

Using constant power excitation, specific heat capacity and density can be measured by analyzing the heater's temperature, if flow speed and thermal conductivity are known. Thermal conductivity is obtained in a certain flow range by evaluating the downstream element utilizing the same excitation mode.

Flow speed can be extracted by knowing the thermal gas parameters and using constant temperature excitation by evaluation of the downstream element.

\section{References}

[1] A. van Putten, S. Middelhoek, "Integrated silicon anemometer", Electronic Letters, Vol. 19, No. 21, 425-426 (1974);

[2] M. Ashauer, H. Glosch, F. Hedrich, N. Hey, H. Sandmaier, W. Lang, "Thermal flow sensor for liquids and gases based on the combinations of two principles", Sensors and Actuators A: Physical, Vol. 73, 7-13 (1998):

[3] D. Reyes, K. Kogan, A. Cubukcu, G. Urban, "Simultaneous flow and thermal conductivity measurement of gases utilizing a calorimetric flow sensor", Sensors and Actuators A: Physical, Vol. 203, 225-233 (2013);

[4] C. Hepp, F. Krogmann, G. Urban, "Determination of thermal gas properties and flow speed using thermal flow sensors", Proc. of the $1^{\text {th }}$ Int. Conference Microfluidic Handling Systems, Enschede, The Netherlands, Oct. 10-12, 91-94 (2012);

[5] D. R. Lide, ed., "CRC Handbook of Chemistry and Physics", $90^{\text {th }}$ edition, CRC Press/Taylor and Francis, Boca Raton, 2010; 